\title{
The Effect of Various Drugs and Inorganic Ions on Bacterial Ribonucleoprotein
}

\author{
By S. DAGLEY, AUDREY E. WHITE AND D. G. WILD \\ Department of Biochemistry, University of Leeds \\ AND J. SYKES \\ Department of Biochemistry, University of Sheffeld
}

In bacteria, protein synthesis is usually accompanied by synthesis of ribonucleic acid (RNA); but in the presence of the antibiotics chloramphenicol (Gale, 1953) and puromycin (Yarmolinski \& de la Haba, 1959; Takeda, Hayashi, Nakagawa \& Suzuki, 1960), or the purine analogues 8-azaguanine (Chantrenne \& Devreux, 1960; Roodyn \& Mandel, 1960) and 8-azaadenine, protein synthesis can be suppressed while RNA continues to be made. Such RNA has often been thought to be abnormal and the cessation of protein synthesis attributed to the fact that it could not function in metabolism. Undoubtedly the RNA formed by Bacillus cereus in the presence of azaguanine is not normal since $23 \%$ of its guanine may be replaced by the analogue (Matthews \& Smith, 1956) mainly at the ends of polynucleotide chains (Mandel \& Markham, 1958). But in the case of chloramphenicol the evidence that the accumulating RNA is abnormal is entirely indirect: thus, this material is unstable while most bacterial RNA is not (Horowitz, Lombard \& Chargaff, 1958); it is broken down by the cells when they are freed from antibiotic and at the same time material absorbing at $260 \mathrm{~m} \mu$ appears in the medium, re-entering the cells when growth resumes (Hahn, Schaechter, Ceglowski, Hopps \& Ciak, 1957); and in ultracentrifuge diagrams for cell-free bacterial extracts it appears as large peaks due to ribonucleoprotein $(\mathrm{RNP})$ sedimenting at 14-18S, whereas in extracts of untreated cells only small peaks are seen in this region of the pattern (Nomura \& Watson, 1959; Dagley \& Sykes, 1959). Chantrenne \& Devreux (1960) have pointed out the similarity between the action of chloramphenicol and that of azaguanine which has been proved to cause accumulation of abnormal RNA : both compounds inhibit synthesis of cytoplasmic protein but not of RNA and neither interfere with the synthesis of cell walls of $B$. cereus. Nevertheless, chemical analysis does not support the view that 'chloramphenicol RNA' is abnormal since the base ratios are those of normal bacterial RNA (Horowitz et al. 1958; McQuillen, 1961). Moreover, if we regard 'chloramphenicol RNA' as a species of RNP which is normally present at much lower concentrations in bacteria as an intermediate stage in ribosome synthesis, most of its abnormal properties can be accounted for; and we shall show that in the analytical ultracentrifuge although it is quite different from 'azaguanine RNA' it does in fact resemble the RNP formed by a mutant which appears unable to stabilize ribosomes due to its inability to synthesize the protein required.

The work of Horowitz et al. (1958) shows that it is not correct to regard the cells as accumulating a type of RNA which, as soon as the antibiotic is removed, they break down and eject. When 'chloramphenicol RNA' was labelled with ${ }^{14} \mathrm{C}$-adenosine it was shown to break down at the same time as it accumulated since the label 
could be 'chased out' with excess of non-labelled nucleoside; no label was lost from cells that had not been treated with chloramphenicol. These and other experiments show that the accumulation of RNA must be regarded kinetically: as soon as chloramphenicol is added, the rate of breakdown is reduced below that of synthesis and RNA accumulates; and when chloramphenicol is removed, breakdown outstrips synthesis and the additional RNA disappears. In tending to stabilize the RNA it is clear from the results of Horowitz et al. (1958) that the chloramphenicol does not act alone, because the RNA which accumulates when it is first added is more stable than that accumulating later, as though some material which is involved in stabilization is in limited supply and becomes progressively exhausted. Since chloramphenicol inhibits protein synthesis, it may well be that this material is protein of the type associated with ribosomes; this may confer stability on ribosomal RNA either by coating it or by imposing upon it a configuration less vulnerable to enzymic attack. Nomura \& Watson (1959) and Dagley \& Sykes (1960) isolated 'chloramphenicol RNA' and showed it to be ribonucleoprotein containing less protein than ribosomal RNP. On this view, accumulation of RNA is a consequence of, and not the reason for, cessation of protein synthesis. When formed inside uninhibited cells this RNA would immediately take part in further reactions, including union with protein, that would lead to stable ribosomes. For a reason which is not evident the antibiotic appears to inhibit breakdown of RNA when the amount of protein available is not sufficient for this purpose. Direct experimental evidence for these views was obtained by Aronson \& Spiegelman (1958) who reported that breakdown of 'chloramphenicol RNA' did not occur when amino acids were present in the culture: instead, this RNA appeared to be converted straight into ribosomal RNA because it was found that ${ }^{14} \mathrm{C}$-amino acids were incorporated into particlebound protein without any simultaneous incorporation of labelled nucleic acid precursors into RNA. Although few details were given of this important experiment, its interest increases as more attention is focused upon intermediate steps in the bacterial synthesis of RNP. By examination of cell-free extracts in the analytical ultracentrifuge we have shown that peaks at 14-18S due to 'chloramphenicol RNP' disappear within $30 \mathrm{~min}$. when cells are suspended in a medium containing amino acids which is free from antibiotic, and that this disappearance is accompanied by a marked enhancement of the peak due to $30 S$ ribosomes. A precursor-product relationship is also suggested by the fact that $30 S$ ribosomes disappear as 'chloramphenicol RNP' accumulates (Pardee, Paigen \& Prestidge, 1957; Dagley \& Sykes, 1959).

Three main stages are discerned in current theories of protein biosynthesis: (1) conversion of an amino acid to an adenylate, (2) its reaction with a suitable 'transfer RNA' molecule to form an aminoacyl-transfer RNA, (3) reaction of this ester, in the presence of guanosine triphosphate and a labile 'transfer factor' (Nathans \& Lipmann, 1961), with a ribosome which may contain 'messenger RNA' whereby the amino acid becomes incorporated into protein. Demoss \& Novelli (1956), Lacks \& Gros (1960) and others have shown that chloramphenicol does not inhibit stages (1) and (2) and may block stage (3) at some point. Yarmolinski \& de la Haba (1959) have drawn attention to the close similarity in structure between the antibiotic puromycin and aminoacyl-transfer RNA-and they showed experimentally that the antibiotic also inhibits stage (3). This was confirmed by Nathans \& Lipmann (1961) who found that neither transfer RNA nor transfer factor were 
affected by puromycin but that ribosomes were irreversibly poisoned for in vitro experiments. Like chloramphenicol, puromycin inhibits the synthesis of protein but not of bacterial RNA which therefore accumulates but is said to differ from 'chloramphenicol RNA' in being stable, i.e. it is not broken down when the bacteria are freed from puromycin and suspended in mineral salts medium (Takeda et al. 1960). We have examined cell extracts of Escherichia coli inhibited with puromycin and find changes in the ultracentrifuge patterns similar to those due to chloramphenicol. Thus, as RNA accumulates large peaks appear, due to material sedimenting at 14-18S; but by contrast, when cells were resuspended in a growth medium free from antibiotic the peaks disappeared much more slowly from ultracentrifuge diagrams of cell extracts and there was no rapid increase in concentration of $30 \mathrm{~S}$ ribosomes. However, chloramphenicol and puromycin evidently act in similar fashions; and the observation that accumulating RNA may be rapidly degraded by the cells in one case and not in the other is not convincing evidence that there are two types of RNA which differ in structure. We found that resumption of protein synthesis in a puromycin-free growth medium was initially very slow and gradually accelerated. By virtue of its similarity in structure, puromycin may become firmly attached to sites normally occupied by transfer RNA and it may be displaced from them only when the cell is able to build up certain concentrations of metabolites. If the material sedimenting in cell extracts is precursor RNA for ribosomes, stabilized at this point by combining with puromycin, it may differ from 'chloramphenicol RNA' only in the rate at which the antibiotic is displaced from combination. If this should occur slowly, the RNA may be incorporated directly into stable ribosomes rather than suffer degradation before they are synthesized.

We have examined cell-free extracts of Bacillus cereus prepared $50 \mathrm{~min}$. after addition of $40 \mu \mathrm{g}$. azaguanine $/ \mathrm{ml}$. culture; this caused marked inhibition of growth as well as an increase in the ratio $\mathrm{RNA} /$ protein, determined chemically for $1 \mathrm{ml}$. of extract, from 0.28 to 0.37 . Extracts were also prepared 45 and 85 min. after guanosine had been added to reverse the action of the analogue and allow growth to resume. For none of the extracts was there evidence that materials had been produced similar to that giving rise to the 14-18 $S$ peaks which result from poisoning the cells with chloramphenicol or puromycin. The only changes observed in patterns were due to a marked increase in concentration of $30 S$ ribosomes when azaguanine had been displaced. Similar results were obtained with cultures of Escherichia coli treated with aza-adenine. This difference in rates of sedimentation of the RNA which is synthesized in the presence of chloramphenicol or azaguanine stands in contrast to various similarities in action already noted (Chantrenne \& Devreux, 1960); however 'azaguanine RNA' has been proved to be chemically abnormal whereas 'chloramphenicol RNA' has not. Mandel \& Markham (1958) found that the polynucleotides built during inhibition with azaguanine are relatively short, and from our work we conclude that they sediment slower than $14 S$; nevertheless, after azaguanine has been ejected $30 S$ ribosomes may be synthesized from these additional polynucleotides at a speed sufficient to cause an increase in ribosome concentration above the normal value.

Borek \& Ryan (1958) showed that RNA continued to be made by a mutant of Escherichia coli requiring methionine when protein synthesis had ceased due to 
lack of methionine. Dr W. Hayes kindly supplied us with a similar strain of $E$. coli which showed this effect. This mutant synthesized little or no protein when cells were suspended in a methionine-free medium and after $180 \mathrm{~min}$., synthesis of additional RNA also ceased. Cell-free extracts made at this time showed additional peaks in about the same region of the ultracentrifuge pattern as those due to "chloramphenicol RNP', namely at $14 S$ and $16 S$. When methionine was added to restore protein synthesis these peaks diminished and there was a simultaneous increase in area of the peak due to $50 S$ ribosomes: this occurred over a period in which the increase in cell mass was equivalent to less than a third of a generation. These peaks at $14 S$ and $16 S$ may thus be due to ribosomal precursor material stabilized by the protein known to be 'turning over' in starved cells (Mandelstam, 1960); but proof of this must await the results of tracer experiments designed to decide whether the material is incorporated directly into the $50 S$ ribosomes, as the ultracentrifuge results suggest, or whether it is first broken down and the ribosomes then resynthesized. Meanwhile it has been observed that the RNA accumulated by the

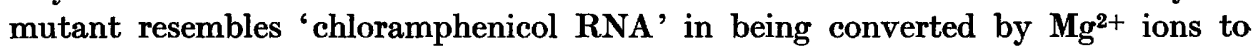
larger, or at least more rapidly sedimenting, ribosomes and also in being rapidly broken down to smaller particles on treatment with sonic vibrations.

For all our experiments the cell-free extracts were made with water or phosphate buffer $(0.015 \mathrm{M}$ or $0.066 \mathrm{M})$ or tris buffer $(0.001 \mathrm{M})$ and all gave essentially the same ultracentrifuge pattern when used to extract disrupted bacteria from the same crop. We did not make a practice of extracting with solvents containing 0.01 M-magnesium acetate, such as other workers have used, for the following reasons: (1) there is no evidence that the $70 S$ ribosomes which are formed at this elevated concentration, and which readily dissociate when it is lowered, are those ribosomes which take part in protein synthesis (Tissières, Schlessinger \& Gros, 1960) or indeed in any metabolic process; (2) on the other hand there is direct evidence that, in the intact bacterial cell, readily-dissociated $70 S$ ribosomes are normally present at much lower concentrations than other RNP particles (Bowen, Dagley, Sykes \& Wild, 1961); (3) added magnesium acetate would have aggregated most of the material that we suggest may be precursors of ribosomes, and changes in ultracentrifuge patterns which appear to be meaningful would not have been observed.

There are two other features of this work which appear relevant to current problems. First, the rapid disappearance of additional RNA following removal of chloramphenicol, azaguanine or azaadenine was accompanied by a rapid rise in concentration of $30 S$ ribosomes but not, in the first instance, of $50 S$ ribosomes. Resumption of protein synthesis by the methionine-requiring mutant took place with a loss of the 14-16S peaks and a rise in concentration of $50 S$, but not $30 S$ ribosomes. Although, therefore, $50 S$ and $30 S$ ribosomes may have common precursors they appear to be formed from them by separate and distinct metabolic routes. Second, if the peaks at 14-16S shown by extracts of the mutant are indeed due to ribosome precursors then this RNA would become labelled rapidly in cells exposed to a suitable radiotracer; and on raising the $\mathrm{Mg}^{2+}$ ion concentration of the extract it would sediment at $30 S$ or adhere to larger ribosomes. Such material would therefore behave similarly to the 'messenger RNA' found in uninfected bacteria by Gros et al. (1961) and is clearly not readily distinguished from it solely by sedimentation studies. 
We wish to thank the Medical Research Council for the provision of a Research Assistantship for A.E.W.

\section{REFERENCES}

Aronson, A. I. \& Spiegelman, S. (1958). On the use of chloramphenicol-inhibited systems for investigating RNA and protein synthesis. Biochim. biophys. Acta, 29, 214.

Borek, E. \& Ryan, A. (1958). Studies on a mutant of Escherichia coli with unbalanced ribonucleic acid synthesis. 2. The concomitance of ribonucleic acid synthesis with resumed protein synthesis. $J$. Bact. $75,72$.

Bowen, T. J., Dagley, S., Sykes, J. \& Wild, D. G. (1961). Nature of the ribosomes present in bacteria and yeast: a re-appraisal. Nature, Lond. 189, 638.

Chantrenne, H. \& Devreux, S. (1960). Action de la 8-azaguanine sur la synthèse des protéines et des acides nucléiques chez Bacillus cereus. Biochim. biophys. Acta, 39, 486.

Dagley, S. \& Sykes, J. (1959). Effect of drugs on components of bacterial cytoplasm. Nature, Lond. 183, 1608.

DAGLEY, S. \& SyKeS, J. (1960). Bacterial ribonucleoprotein synthesized in the presence of chloramphenicol. Biochem. J. 74, 11 P.

Demoss, J. A. \& Novelli, D. (1956). An amino acid dependent exchange between ${ }^{32} P$ labelled inorganic pyrophosphate and ATP in microbial extracts. Biochim. biophys. Acta, 22, 49.

GALE, E. F. (1953). Assimilation of amino acids by Gram-positive bacteria and some actions of antibiotics thereon. Advanc. Protein Chem. 8, 285.

Gros, F., Hiatt, H., Gilbert, W., Kurland, C. G., Risebrough, R. W. \& Watson, J. D. (1961). Unstable ribonucleic acid revealed by pulse labelling of Escherichia coli. Nature, Lond. 190, 581.

Hahn, F. E., Schaechter, M., Ceglowski, W. S., Hopps, H. E. \& Ciak, J. (1957). Interrelations between nucleic acid and protein biosynthesis. 1. Synthesis and fate of bacterial nucleic acids during exposure to, and recovery from, the action of chloramphenicol. Biochim. biophys. Acta, 26, 469.

Horowitz, J., Lombard, A. \& Chargaff, E. (1958). Aspects of the stability of a bacterial ribonucleic acid. $J$. biol. Chem. $233,1517$.

LACKS, S. \& Gros, F. (1960). A metabolic study of the RNA-aminoacid complexes in Escherichia coli. J. mol. Biol. 1, 301.

Mandel, H. G. \& Markham, R. (1958). The effects of 8-azaguanine on the biosynthesis of ribonucleic acid in Bacillus cereus. Biochem. J. 69, 297.

MANDELSTAM, J. (1960). The intracellular turnover of protein and nucleic acids and its role in biochemical differentiation. Bact. Rev. 24, 289.

Matthews, R. E. F. \& Smith, J. D. (1956). Distribution of 8-azaguanine in the nucleic acids of Bacillus cereus. Nature, Lond. 177, 271.

McQuillen, K. (1961). Protein synthesis in vivo: the involvement of ribosomes in Escherichia coli. In Protein Biosynthesis, p. 263, ed. by R. J. C. Harris. London: Academic Press.

Nathans, D. \& LipmanN, F. (1961). Aminoacid transfer from aminoacyl-ribonucleic acids to protein on ribosomes of Escherichia coli. Proc. nat. Acad. Sci., Wash. 47, 497.

Nomura, M. \& WATson, J. D. (1959). Ribonucleoprotein particles within chloromycetininhibited Escherichia coli. J. mol. Biol. 1, 204.

Pardee, A. B., Paigen, K. \& Prestidge, L. S. (1957). A study of the ribonucleic acid of normal and chloromycetin-inhibited bacteria by zone electrophoresis. Biochim. biophys. Acta, 23, 162.

Roodyn, D. B. \& MANDEL, H. G. (1960). The differential effect of 8-azaguanine on cell wall and protoplasmic protein synthesis in Bacillus cereus. J. biol. Chem. 235, 2036.

Takeda, Y., HaYashi, S.-I., Nakagawa, H. \& Suzuki, F. (1960). The effect of puromycin on ribonucleic acid and protein synthesis. J. Biochem. (Tokyo), 48, 169.

Tissières, A., Schlessinger, D. \& Gros, F. (1960). Amino acid incorporation into proteins by Escherichia coli. Proc. nat. Acad. Sci., Wash. 46, 1450.

YARMolinski, M. D. \& DE LA HABA, G. L. (1959). Inhibition by puromycin of amino acid incorporation into protein. Proc. nat. Acad. Sci., Wash. 44, 189. 